\title{
Overview of Some Biomedical Research Projects in Tropical Medicine Conducted at the Instituto Venezolano de Investigaciones Cientificas
}

\author{
Egidio Romano $^{+}$, Italo Cesari, Ananias Escalante, Ferdinando Liprandi, \\ Jose Antonio O'Daly, Hilda Perez, Howard Takiff
}

Instituto Venezolano de Investigaciones Cientificas, Apartado 21827, Caracas 1020A, Venezuela

The Instituto Venezolano de Investigaciones Cientificas (IVIC) is a government-funded multidisciplinary academic institution dedicated to research, development and technology in many areas of knowledge. Biomedical projects and publications comprise about $40 \%$ of the total at IVIC. In this article, we present an overview of some selected research and development projects conducted at IVIC which we believe contain new and important aspects related to malaria, ancylostomiasis, dengue fever, leishmaniasis and tuberculosis. Other projects considered of interest in the general area of tropical medicine are briefly described.

This article was prepared as a small contribution to honor and commemorate the centenary of the Instituto Oswaldo Cruz.

Key words: ancylostomiasis - dengue - malaria - tuberculosis - Instituto Venezolano de Investigaciones Cientificas

The Instituto Venezolano de Investigaciones Cientificas (IVIC), originally founded in 1955 as the Instituto Venezolano de Neurología e Investigaciones Cerebrales, was organized in 1959 as a multidisciplinary research institution financed mainly by public funds. Until 1999, it was ascribed to the Ministry of Health being now ascribed to the recently created Ministry of Science and Technology. Research, development and technology are conducted at IVIC mainly in biomedical areas, about $40 \%$ of the total, and also in chemistry, physics, mathematics, ecology and anthropology. A post-graduate school having programs leading to the master and $\mathrm{PhD}$ degrees, is also functioning. A center for technology is in charge of specialized services. Additionally since 1997, IVIC runs a human plasma fractionation industrial facility producing serum albumin, gammaglobulins and Factor VIII. IVIC's scientific library is one of the largest and most complete in Latin America receiving about 4,600 titles and serving more than 500,000 consultations/year. IVIC output in terms of scientific publications is about 300 articles/year

\footnotetext{
${ }^{+}$Corresponding author. Fax: +58-2-504.1428. E-mail: eromano@ivic.ve

Received 7 August 2000

Accepted 4 September 2000
}

most of them in internationally refereed SCI-listed journals. Since 1973 to now, the Center for Advanced Studies has graduated 750 Master/PhD students.

Because of the special interest in this symposium in topics related to tropical medicine, we have selected for a brief presentation some research projects dealing with aspects related to malaria, schistosomiasis, dengue, leishmaniasis, and tuberculosis. However, many others relevant investigations in the field, some of which will be only mentioned under the heading of "other research projects related to tropical medicine carried out at IVIC".

\section{MALARIA}

New knowledge in malaria and its influence on the development of new control strategies (contributed by Hilda Perez).

Few years ago, the World Health Organization (WHO) promoted the aim of health for all by the year 2000. Nowadays at the beginning of the millenium, this goal seems to be still far away for the countries in development, on the contrary the situation of several endemies has worsened in relation to the 60's. A good example for this opinion is the situation of malaria, which was considered in the 60's "eradicated" or under control in many Latin American countries and which presently has returned stronger than in the past. Therefore we face an old problem for which we hope to find future solutions with new ideas and technologies. 
Chemotherapy and steps that interfere with the contact man-vector, were and are two big pedestals in the strategy of fighting malaria. However, the geographic expansion of Plasmodium falciparum resistant to cloroquine and other antimalarials and of mosquitoes which evade the action of insecticides are dilemmas of today science for which are expected solutions coming from tomorrow science.

During the last 15 years, considerable efforts have been made to develop malaria vaccines. DNA recombinant technology has been used to identify parasite molecules with protective potential assuming the hypothesis, which emphasize the importance of surface molecules of the parasite's extracellular stages. A list of prototype vaccines is in the agenda of the WHO waiting to be evaluated in the immediate future. However, studies performed in endemic regions are indispensable to understand the dynamic of the immune response to these molecules in the populations exposed to the transmission. For example, studies on the molecular epidemiology of the CSP and MSP-1 of P. vivax in the Southeast of Venezuela, promoted by IVIC, indicates the existence of polymorphic variants which have to be considered for the regional benefit of potential vaccines based in subunits of these molecules.

How and why Plasmodium parasites transport protein and other macromolecules of parasite origin from the cytoplasm of the parasite to the cytoplasm of the erythrocyte and eventually to it surface membrane, from the organelle which needs to establish in a cell lacking the machinery of molecular transport, the molecular pathway and gene expression of a group of genes which are necessary for the development of the erythrocytic phase, are possibly important aspects to be considered for the rational design of new drugs. In P. vivax which cause more than $70 \%$ of malaria morbidity in Latin America, we have found a system of intracellular traffic related to the secretion of specific parasite proteins which are necessary for the survival of the parasite in the erythrocyte. This type of studies becomes very important since new evidence indicate the emergency of $P$. vivax resistant to cloroquine in some regions of the globe.

Operational investigations which take into account the local and regional particularities of the epidemiology of malaria and which evaluates the strategies of malaria control are necessary in malaria endemic regions as long as malaria is a public health problem. In this context investigations promoted by IVIC are carried out to evaluate the therapeutic efficacy of cloroquine for the treatment of $P$. vivax malaria, giving favorable results for the therapeutic scheme recommended by the WHO. Also, the application of molecular biology meth- ods for the diagnosis of malaria in Southeast Venezuela have proved the usefulness of these technologies for the diagnosis of malaria. The studies have shown that there is an important underestimation of therapeutic failures, and also of the incidence of mixed infections with $P$. vivax and $P$. falciparum, multiple infections with $P$. vivax, $P$. falciparum, and $P$. malariae, a high rate of infection with $P$. malariae in the indigenous population of the Amazon. The long lasting infections with $P$. malariae is an enigma in the biology of this parasite and so is the possible relation with endemic pockets yet to be localized in the selvatic regions of the Amazon.

The benefits that mankind hopefully can derive from new knowledge and technologies to fight tropical disease goes beyond the laboratories. For instance, it is quite possible that in ten years time new and better drugs and vaccines are available for several tropical diseases; even so, at this, time difficult decisions have to be taken to make sure that these benefits will reach the needed population.

Evolutionary biology of malaria parasites (contributed by Ananias Escalante; work done in collaboration between IVIC, Caracas and the Centers for Disease Control and Prevention, Atlanta)

It is estimated that 2300 million people $41 \%$ of the world population live in regions at risk for malaria. Malaria infection has been estimated between 300-500 million cases/year with 1.5 to 2.7 associated deaths, $95 \%$ occurring in sub-Saharan Africa. At least four species of Plasmodium causes the disease being $P$. falciparum, and vivax more important and $P$. malariae and $P$. ovale the other.

We want to estimate the factors influencing the rate of evolution of the antigens which are considered good candidates for a malaria vaccine. These proteins are under a positive natural selection, that is, selection maintains and determine the accumulation of genetic variability. The problem is to obtain a reliable alignment which allows comparison of homologous regions in proteins of great variability. Generally, the algorithm of alignment uses a phylogenetic tree as a guide that is estimated by comparing two by two in all the sequences. This tree is then used as a guide to generate the alignment of multiple sequences. Without alignment it is not possible to identify homologous regions and evaluate rate of evolution. It is difficult to establish homologies at priory if selection is acting on the proteins. It would be ideal to use an independent tree, generated on the basis of information different of the antigen under study, as a guide to align the antigens. We are currently studying the phylogenetic relationship of the Plasmodium ge- 
nus based on five genes in three genomes. This will allow us to construct an independent tree for the study of the rate of evolution in terms of antigens. A second factor to consider for the estimation of the rates of evolution is to incorporate information on the structure and chemical properties of the amino acids. For this purpose, we are modeling the 3D structure of the proteins and exploring the amino acids substitution on the computed structure. The methodologies used for this approach are as follows: (1) homologous sequences with at least a $40 \%$ identity are used; (2) the average structure of all possible models is generated; (3) the best structure, the one which requires less energy, is then modeled by computer analysis. Applying this method, it can be seen that structural changes also imply changes in the polar requirement of the amino acids of the proteins as shown in the analysis of the window. The results are then incorporated in the estimation of the rates of evolution.

Genetic polymorphism studies in P. falciparum - What is the variability of the antigens that are being used in the current vaccine candidates? We have found that at least for three different antigens there is a greater variability in Africa. In general also, can be concluded that the variability found in Venezuelan isolates is less pronounced when compared with similar samples from Asia and Africa.

In conclusion, from these types of studies has been generated data on antigens that could be incorporated in vaccines for $P$. falciparum, and $P$. vivax. There is abundant evidence, which indicates that natural positive selection is important in the conservation of polymorphism.

\section{SCHISTOSOMIASIS}

Immunoepidemiological studies (contributed by Italo Cesari and Diana Ballen)

Schistosoma mansoni has been endemic in Venezuela probably since its introduction with the slave trade, in the XVI century. The disease is transmitted by Biomphalaria glabrata snails; the endemic zone comprises $15,000 \mathrm{~km}^{2}$ representing $1.6 \%$ of the national territory where more than $40 \%$ of the population lives. In 1943, the Venezuelan Ministry of Health organized an integrated Schistosomiasis Control Program (SCP) based on snail vector elimination, health education, environmental and sanitation improvement, epidemiological surveillance, coprologic diagnosis, and medical treatment. These measures decreased the coprologic prevalence from about $14.6 \%$ (19431960 ) to $1.7 \%$ in the 80 's. Transmission became low and highly focal and, in general, more than $70 \%$ of the stool-positive individuals excreted less than 100 epg of feces. Because of the success of the control strategies, sanitary authorities assumed, however, that schistosomiasis was disappearing. Nonetheless, after the reactivation of a few old foci, some researchers realized that schistosomiasis was being underestimated: coprological techniques were not sensitive enough to detect light infections and immunological assays then became important alternative tools for the detection of asymptomatic and chronic schistosomiasis. As a consequence, schistosomiasis research at IVIC (going on since 1973), was partially redirected to solve the diagnostic problem by developing a sensitive and specific immunodiagnostic test.

Development of the alkaline phosphatase immunoassay (APIA) - In 1987, Cesari et al. reported the antigenicity of some adult worm and egg enzymes and suggested their possible use as antigens for immunodiagnosis. The antigenicity of a membranebound, adult worm alkaline phosphatase (ALP) was confirmed, and a simple solid phase assay to detect anti-ALP IgG antibodies in serum was developed. The method exploits the intrinsic catalytic activity of the ALP antigen to reveal the immunocapture event. In well-defined conditions, APIA showed high sensitivity (> 89\%) and specificity (100\%), as compared to parasitological techniques.

Field immunoepidemiological studies with APIA - In 1997-1999, several immunoepidemiological surveys were conducted by the SCP and the Schistosomiasis Research Group (people from different academic and scientific Venezuelan institutions) in different rural and sub-urban endemic communities. The surveys were performed by looking at the presence of $S$. mansoni eggs in the stools (Kato-Katz technique) and by applying four different serologic tests. Anti-egg response was assessed by the circumoval precipitin test (COPT) of Oliver-González, and a modified ELISA originally described by Voller, based on $S$. mansoni soluble egg antigen (SEA) pretreated with sodium metaperiodate. Anti-adult antibodies were determined by APIA and Western Blot (WB), both performed with a $n$-butanol extract of the adult worm membrane fraction. WB was considered positive when sera reacted specifically with any one of a characteristic group of S. mansoni membrane antigens ( 10-110 kDa). Agreement between tests was assessed by the Cohen's kappa ( $k$ ) coefficient.

In a recently reactivated old transmission site, with moderate intensity of infection (up to $300 \mathrm{epg}$ of feces), APIA exhibited, as compared to the COPT, high sensitivity $(91.1 \%)$ and specificity (93.8\%), high $k$ agreement (0.77), and prevalence (19.3\%), similar to that detected by COPT (15.4\%). In a low transmission site, APIA showed good sensitivity (86.8\%) and specificity (84.2\%), but lower 
agreement $(k=0.43)$, and a higher prevalence (24\%) than COPT (11.6\%). Surprisingly, considerable seropositivity was found in a past endemic area without current transmission, treated more than 15 years ago. In this area, APIA showed high sensitivity (92.7\%), but lower specificity $(77.5 \%)$, low $k(0.49)$ and high prevalence $(33.9 \%)$ as compared to COPT (16.1\%). Compared to ELISA, APIA showed in most regions studied, low sensitivity (60.9-67.3\%) and high specificity (88.9$95.9 \%)$, similar prevalence and $k$ (0.56 to 0.62 ). The highest $k$ agreements were obtained between APIA and WB ( $k 0.86$ to 0.90$)$, suggesting that the two assays were mostly detecting the same cases. The patterns of positive bands varied with different sera, and were generally more complex in samples from moderate transmission sites. Based on $k$ values, APIA, ELISA, and COPT were probably not detecting the same cases, particularly in low transmission sites.

The ELISA prevalence was higher than that by COPT in all sites studied, suggesting the existence of numerous COPT-negative/ELISA-positive individuals. The anti-egg response (ELISA, COPT) was more important in sites with current transmission, whereas the anti-adult response (APIA) decreased from past to moderate transmission sites, suggesting the increased existence of individuals infected with a low number of adult parasites or unisexual infections in past or low transmission sites. In recently treated transmitting areas, seropositivity by all the tests may be due to the presence of remaining circulating anti-adult and/or anti-egg antibodies, drug-tolerant worms, and/or reinfection. However, in old-treated, past transmission sites, it is unlikely that cured individuals still have circulating anti-ALP antibodies, and COPT-negative/ APIA positive individuals found in these sites are probably lightly-infected individuals that were never treated and still have few long-lived schistosomes (low levels of egg excretion) that continuously stimulate an anti-adult membrane response (WB positiveness).

In conclusion, APIA was proven to be a useful serological test to specifically detect the $S$. mansoni infection in medically untreated endemic areas. Diagnosis by APIA (anti-adult) and by ELISA (anti-egg) gives complementary information and the two tests should be used to have a better epidemiological assessment of those areas and to select individuals for medical treatment.

\section{DENGUE}

Genetic diversity of dengue 1 viruses (contributed by F Liprandi, AP Goncalvez, F Pujol, JE Ludert, from IVIC and R Salas, D Tovar, from Instituto Nacional de Higiene, INH)
Dengue fever (DF) and dengue hemorragic fever/dengue shock syndrome (DHF/DHSS) are probably the most important mosquito-borne viral diseases-affecting humans worldwide. The spectrum of disease is caused by dengue viruses that occur as four antigenically-different serotypes (from 1 to 4). Infection with one serotype of dengue virus protects against the homologous but not against the heterologous reinfection.

One of the most challenging aspects of dengue biology has been the identification of the factors and the underlying mechanisms causing an infection with dengue virus to evolve toward the more severe hemorragic syndrome. One established risk factor for the hemorragic form is a secondary infection by a dengue serotype different from the one associated to the primary infection. More recently it has become apparent that some genetic variants of dengue virus are more virulent than others, that is, they have the potential to produce DHF.

In Venezuela dengue has become in the last decade one of the leading problems of public health. For example, in the last year (1999), 27,000 cases of dengue have been reported, and of these approximately $10 \%$ correspond to the more severe hemorragic form.

The history of dengue in Venezuela has been similar to the one of the rest of the Caribbean region. Dengue occurred sporadically, associated to different serotypes, until 1977, when dengue serotype 1 was introduced into the American continent and produced important epidemics of dengue in several countries. Venezuela was hit in 1978. Despite thousands of dengue cases, no hemorragic forms were reported until 1989 when the first epidemic of hemorragic dengue occurs in Venezuela, associated mainly with dengue serotype 2 .

From 1989 until today, there has been recurring epidemics almost every year. Typically, epidemics have followed a cyclic pattern, with an incidence rate increasing dramatically in association with the rainy season. Also there has been a turnover in the serotype of dengue strains predominating year after year. The simultaneous co-circulation of several serotypes (serotypes 1,2 and 4) is typical of an hyperendemicity situation like the one observed during the last twenty years in Southeast Asia, characterized by a high incidence of hemorragic dengue.

IVIC has recently become involved in dengue research through a collaborative project in which take part scientists from IVIC and INH. The main objective of this line of investigation is the molecular characterization of the dengue virus strains that circulate in Venezuela. Detection of variation within a serotype have become increasingly important in dengue research: genetic studies based 
on the combined use of methodologies like PCR and sequencing have permitted a new understanding of (a) patterns of dengue epidemics, allowing to identify virus strains in a unambiguous way and to follow their movements from one country to another; (b) eventual association of particular strains (or types) of viruses with the more severe forms of the infection (DHF/DSS).

The best examples of the latter situation, namely a strain basis to DHF and DSS, have been the epidemics of hemorragic dengue occurring in the Americas in the 80's (in Cuba in 1981 and Venezuela in 1989 and in the following years). All the hemorragic dengue episodes have been associated to the introduction of dengue serotype 2 strains from Southeast Asia. Remarkably, the circulation of the "American" type virus has been associated only with classic dengue disease.

Sequence analysis of selected regions of dengue genomes has been instrumental to recognize and differentiate genetic variants.

At IVIC, research has focused on the genetic characterization of dengue virus serotype 1 and 4 circulating in the country in the last decade. Particularly interesting is the case of dengue 1 . This serotype was introduced for the first time in the America in 1977 and it has been co-circulating in Venezuela, from the 1989 epidemic, together with dengue 2 and 4 . Serotype 1 has been the predominant circulating serotype in different years (93-9497). This serotype has been associated to both classic and hemorragic dengue.

The genetic diversity of dengue 1 virus strains circulating in the American continent after its introduction in 1977, was evaluated studying the sequences of the $E$ gene, which code for the envelope structural protein of the virus. This protein is associated to adsorption and penetration to cells, and to induction of and reaction with, neutralizing antibodies.

The nucleotide sequences of the complete gene of the envelope protein (1,485 nucleotides: approximately one seventh of the whole genome) of 30 dengue 1 strains from different parts of the world were determined by automated sequencing of PCR products spanning the gene of interest. Of these strains, 18 were isolates from eight American countries (seven from Venezuela, the rest from Brazil, Colombia, Mexico, Peru, Trinidad, Aruba, Jamaica, Grenada) covering a period of 20 years.

\section{CONCLUSIONS}

(1) phylogenetic analysis indicated that dengue 1 strains could be divided into three distinct genotypes; (2) all American strains showed a sequence similarity greater than $97 \%$ and fell into one single genotype. Thus, most probably there has been only one type of dengue 1 circulating in the Americas; (3) genotype III which included all the American strains included also strains from Africa (Nigeria, Ivory Coast) and Asia (Burma), confirming that this genotype is wide spread. More data are required to establish the origin of the introduction. The Venezuelan strains included strains associated to classical or hemorragic fever suggesting that this genotype (III) has the potential capability to cause hemorragic dengue. These data show the type of information that can be obtained combining the clinical data and the classical epidemiological observation coupled with molecular analysis. This virology group at IVIC is now pursuing a more in depth understanding of the evolution of the genome of dengue 1 viruses, through the comparison of the complete genome (approximately 10,800 bases) of representative strains of each genotype.

Additional investigations on dengue at IVIC A group lead by $\mathrm{J}$ Cardier is studying the possible involvement of vascular endothelial cells in hemorragic dengue. Interesting data are coming out on activation of these cells and of cytokine involvement. P Taylor has developed a dengue test for the quick and reliable diagnosis of dengue based on the detection of $\operatorname{IgM}$ and IgG specific antibodies. $\mathrm{R}$ Apitz and others are studying alterations of blood coagulation factors and function in hemorragic dengue. R Walder and others are studying aspects of the molecular epidemiology of dengue virus 1 of Venezuelan isolates form old and recent epidemics.

\section{MULTI-DRUG RESISTANT TUBERCULOSIS} (contributed by Howard Takiff, IVIC)

Tuberculosis (TB) is an ancient disease that modern science has been unable to conquer. In fact, there are now more cases on earth than at any other time in history. Worse yet, the development of multi-drug resistance has created outbreaks that are difficult or impossible to treat. However, technology has been able to contribute to understanding how the bacteria develop resistance, and quicker and cheaper ways to detect resistance. In addition insights into the nature of the bacteria, and the roots of its ability to survive human immune defenses may help to develop a future vaccine superior to BCG.

One of the problems with multi-drug resistant TB is that traditional methods of testing for resistance take 6-12 weeks, during which time the patient can be disseminating the resistant strains to others. In the case of Aids patients with multi-drug resistant $\mathrm{TB}$, the patient is often dead before the results of the sensitivity testing are available. This is all fairly irrelevant for our countries, because traditional sensitivity testing requires that sputum 
specimens be cultured, which is rarely done, and then an expensive and laborious process of sensitivity testing.

Automated culture systems used in the United States and Europe are simply too expensive for most developing countries. To try to develop effective, appropriate alternatives, investigators at IVIC are working together in a Latin American network of TB labs called RELACTB that was founded in a meeting at IVIC in 1995. This network includes basic research and clinical labs from most of the countries in the region, including Brazil. One new technique that RELACTB has been evaluating in multicenter trials uses dyes such as MTT or Alamar Blue that change color in the presence of actively growing cells. Now, instead of having to use multiple culture tubes to test for resistance to different concentrations of each of eight or nine primary and second line anti-tuberculosis drugs, all the testing can be done on one 96 well plate, and the results can be read by eye or with an ELISA reader within 10-14 days. This, or a modification of this technique, could represent a significant advance, and costs less than $10 \$$ in reagents, but it requires a fair amount of labor and a liquid culture of the Mycobacterium tuberculosis isolated from the sputum. In other words, it is too expensive and complicated to be used on specimens from all patients who might possibly have resistant bacteria. One solution would be to have an extremely cheap and simple system to screen for drug resistance, and then just test the positives with the Alamar Blue or other system for a full sensitivity profile to guide treatment. In thinking about such a test, one has to think of what can be done reliably in provincial or regional labs, with a minimum of technical difficulty. This means that PCR, Beacon Probes and exacting hybridizations that can detect single base changes, techniques that are celebrated in the developed world, likely do not enter the realm of the possible for provincial mycobacteriology labs. A promising technique developed by Dr Bill Jacobs in New York is being adapted by researchers at IVIC. A luciferase gene was cloned into a mycobacteriophage, a virus that infects M. tuberculosis. A clinical isolate is innoculated into liquid media bacterial culture in the presence of antibiotics, allowed to grow for 710 days, and then infected with the luciferase containing phage. The substrate luciferin is then added, and if the isolate is resistant to the drug, it makes enough ATP to support the luciferase reaction and light is produced. Light equals resistance. This could be done in ELISA plates in a format very similar to the Alamar Blue test, but would require a sophisticated luminometer to read the results. The work at IVIC is to adapt this system to a simple one dollar, one tube assay that will reliably detect which patients that are infected with resistant isolates and therefore require change in their antibiotic regimen and complete sensitivity studies, perhaps with Alamar Blue. This is possible because when isolates resistant to Rifampicin are generally also resistant to Isoniazid and other drugs, so resistance to Rifampicin serves as a marker for multidrug resistance. The investigators in IVIC are working with Dr Jacobs to adapt the phage system for use with cultures taken directly from sputum, to obtain results within two weeks using nothing more than a polaroid camera in a box.

However, to achieve substantial control over TB world-wide will require, first and foremost, increased political and financial resources to improve health care delivery systems, but a TB vaccine that is much more effective than BCG would also help. Over the past five years many of the known TB antigens have been tried as vaccines using a variety of strategies, but none has proved better than the inadequate BCG. These failures have led to the conclusion that perhaps a more fruitful approach will be to first try to better understand the basic biology of $M$. tuberculosis and why it is an effective pathogen, and then use this knowledge to design an effective vaccine.

One key to the survival of $M$. tuberculosis in the macrophage is its ability to resist the reactive oxygen species produced by the macrophage. One important effect of oxidation is disruption of protein function due to the formation of disulfide bonds. Thioredoxin and thioredoxin reductase constitute key system for keeping the sulfur groups of Cysteine amino acids reduced and their proteins functional. Thioredoxins are present in most organisms from humans to bacteria. In bacteria, there is a developing body of research on how thioredoxin and associated proteins function, but little is know about how they are regulated. A PhD student at IVIC recently completed her thesis studying how $M$. tuberculosis responds to oxidative stress and has identified a transcription factor that regulates the expression of the thioredoxin system in this bacteria. To avoid using the virulent $M$. tuberculosis in the lab, she worked with BCG, the avirulent vaccine strain, and other non-pathogenic mycobacteria. The transcription factor, SigM, is one of several sigma factors in the M. tuberculosis genome. Sigma factors combine with the core RNA polymerase to give it promoter specificity, and many of these induce a protective bacterial response to environmental stimuli. In other words, in the presence of some environmental stress, different sigma factors become active, bind to the core RNA polymerase, and transcribe genes involved in minimizing damage to the bacteria. There are two general ways to regulate such 
a response, increase its expression, or increase its activity. LacZ gene fusions were used to measure the expression of sigma factor, SigM under different stress conditions. It was found to increase with high temperature and also in stationary phase, when the bacteria has exhausted its nutrient source and stops replicating. It has been shown in other bacteria that both, high temperature and stationary phase are accompanied by oxidative stress and formation of disulfide bonds. Curiously, the expression of SigM is not increased in oxidative stress, such as when the bacteria is exposed to hydrogen peroxide or sources of superoxide radicals. However, when a mutant strain was constructed to lack Sigma Factor M, it was found to survive like WT bacteria at high temperature and in stationary phase, but was unable to survive oxygen stress. But now what could be the genes normally regulated by SigM that are not induced in the sigM mutant? It turns out that while thioredoxin activity normally increases in oxidative stress, in the SigM mutant, thioredoxin activity does not increase above baseline in oxidative stress. Thus SigM does not regulate baseline thioredoxin expression, but is responsible for its induction in oxidative stress as an important defense mechanism. But how can this occur if the expression of SigM does not increase in oxidative stress? One possibility is that SigM is regulated by an anti-sigma factor whose gene lies just downstream of SigM in the chromosome. Using a lacZ fusion to the SigM promoter integrated into the chromosome of BCG, the student transformed the strain with plasmids containing either the vector alone, SigM alone, or SigM plus the downstream gene. The multicopy plasmid with SigM increased expression, so SigM exhibits positive autoregulation. However, the presence of the adjacent open reading frame diminished the effect of the SigM, suggesting that it is a negative regulator. Likely it functions like anti-sigma factors found adjacent to other sigma factors, it binds to and inactivates the sigma factor, until some environmental condition, such as oxidative stress, somehow changes its conformation so that it no longer binds to SigM. The sigma factor is then free to bind to the RNA polymerase and transcribe the genes it regulates.

While the response to stress in $M$. tuberculosis is complex and involves several sigma factors with overlapping responsibilities, SigM appears to play a crucial role in the response to oxidative stress. So, SigM may be critical for M. tuberculosis virulence, which is the ability to survive and multiply within the inhospitable macrophage. This work should lead to a better understanding of the nature of this virulence, and perhaps to a successful vaccine strategy, or the development of new and novel therapeutic agents.

\section{LEISHMANIASIS}

Vaccine follows a summary of studies done by JA O'Daly and his team reported in Gac Med Caracas (1995 103: 133-179).

In the "Valle Arriba" neighborhood located in the outskirts of the town of Guatire, Miranda State, at one hour by road east from Caracas, with a morbidity of 24.76 per thousand, we have applied an amastigote vaccine against leishmaniasis in 3,191 people with a follow up period of three years. We have also applied the vaccine in the adjacent rural zone "El Ingenio" hyperendemic for leishmaniasis with a morbidity of 162.04 per thousand.

The vaccine contains 8-10 proteins from four leishmania species after treatment of amastigotes with TLCK and NP40.

Before the vaccine application in Valle Arriba, both the vaccinated and the control unvaccinated group showed a $24 \%$ positivity in the intradermal reaction (IDR) with the antigen of the vaccine. One month after three doses of the vaccine 95.67 of the vaccinees previously IDR negative showed conversion to a positive IDR. The IDR in both the prevaccinated IDR-negative and IDR-positive vaccinees has increased every year for three consecutive years up to $20 \mathrm{~mm}$ diameter without new doses of the vaccine.

The follow up for three years in house by house visit reveals a higher rate of infection in the nonvaccinated than in the vaccinated group, both living in the same house and exposed to the same infection factors. The morbidity rate was 7.94 per thousand in the vaccinated group, while it reached 56.17 in the control-unvaccinated group, an incidence 7.07 times bigger after three year of the field trial.

In the community of "La Planta", a hyperendemic for leishmaniasis, $41.8 \%$ of the population showed a positive IDR before vaccination. The disease increase during 1991-1994 when 82 cases of leishmaniasis were reported while in the previous years were diagnosed only 1-2 cases per year. In total, from 1972 to 1994,122 cases have been diagnosed in this community. The highest morbidity (205.23) per thousand was found in the age 0-5 years old, which points out to intradomiciliary transmission of the disease. The pre-vaccinated IDR negative group showed 95-100\% conversion to IDR positive with a progressive increase of the response of the IDR after each doses of the vaccine. In La Planta the negative pre-vaccinated IDR people showed $15.3 \%$ of leishmaniasis in the vaccinated group and $58.8 \%$ in the control-unvaccinated group, a significant difference. The odds ratio in non vaccinated was 3.6 and in vaccinated 0.274 , a value less than 1 which speaks about the efficiency of the vaccine. 
No difference was observed nor in the ELISA or in the immunoblotting pattern of sera before and after vaccination which points out that the immunological reaction after the vaccine is not humoral but mainly dependent on cellular immunity.

By 1999, a total of 10,000 people have been vaccinated and a follow up is almost complete.

O'Daly has found that in the endemic region of Valle Arriba the vaccine confers a protection of $85.9 \%$ and $71 \%$ in the endemic region La Planta. Epidemiological follow up in these regions, in the period 1995-1998, have shown new cases of leishmaniasis only to occur in the non-vaccinated group.

Oddly enough, O'Daly has found that his leishmania vaccine seems to be an excellent therapy for psoriasis. By the end of 1999, 2,650 patients had been treated showing a 60-70\% clinical remissions of the lesions.

Another investigation on leishmaniasis at IVIC is carried out by M Lampo. She is trying to identify wild animals species from which feeds Lutzomyia longipalpis, the main vector of visceral leishmaniasis, a very severe form of leishmaniasis in America.

OTHER RESEARCH PROJECTS RELATED TO TROPICAL MEDICINE THAT ARE CARRIED OUT AT IVIC

\section{Rotavirus and diarrhea}

F Michelangeli, F Liprandi and others study the mechanism of penetration of the virus in the cell and the role of intracellular $\mathrm{Ca}++$ in the viral cycle. The group also works on the molecular epidemiology of rotavirus causing diarrhea in man and domestic animals.

\section{Hepatitis C Virus}

F Pujol and others are evaluating the use of synthetic peptides corresponding to molecules of the viral capside in the diagnosis of Hepatitis C
Virus. Similar studies are also going on for the diagnosis of Hanta virus.

\section{Chagas disease}

J Urbina is leading a team working on the development of therapeutic drugs for this disease. Triazol compounds, by inhibiting the synthesis of ergosterol in Trypanosoma cruzi, have shown a strong anti-proliferative activity for the parasite as well as for pathogenic fungi. The investigation is well advanced, is receiving strong support not only from IVIC but also from the WHO and recently from the Howard Hughes Medical Institute. Preclinical studies are complete and field studies are planned to begin soon.

Z Layrisse is studying the possible association between cardiac failure due to chronic Chagas disease and genetic make up.

\section{Paracoccidioides braziliensis}

G Sanblas is studying the biochemical basis for the dimorphism in this pathogenic fungus. In collaboration with a multidisciplinary team an effort is going to rationally design new antifungal compounds.

\section{Scorpionism}

G D'Suze and C Sevcik are studying the toxinology of venom's from Venezuelan scorpions.

\section{Venom from a caterpillar}

CL Piñango and her team have studied for many years now, the severe hemorragic disorder caused by contact with a caterpillar, Lonomia achelous, originally described in Venezuela but also found in Brazil and some others South American countries. The hemorragic syndrome is now biochemically well characterized and a rational treatment has been worked out. 